\title{
A Simplified Comorbidity Evaluation Predicting Clinical Outcomes Among Patients With Coronavirus Disease 2019
}

\author{
Jessica J. Kirby a, c, Sajid Shaikh ${ }^{\text {b, c }}$, David P. Bryant ${ }^{a}$, Amy F. Ho ${ }^{a}$, James P. d'Etienne ${ }^{a}$, \\ Chet D. Schrader ${ }^{\text {a }}$, Hao Wang ${ }^{\text {a, }}$ d(D)
}

\begin{abstract}
Background: Patients with coronavirus disease 2019 (COVID-19) have shown a range of clinical outcomes. Previous studies have reported that patient comorbidities are predictive of worse clinical outcomes, especially when patients have multiple chronic diseases. We aim to: 1) derive a simplified comorbidity evaluation and determine its accuracy of predicting clinical outcomes (i.e., hospital admission, intensive care unit (ICU) admission, ventilation, and in-hospital mortality); and 2) determine its performance accuracy in comparison to well-established comorbidity indexes.
\end{abstract}

Methods: This was a single-center retrospective observational study. We enrolled all emergency department (ED) patients with COVID-19 from March 1, 2020, to December 31, 2020. A simplified comorbidity evaluation (COVID-related high-risk chronic condition (CCC)) was derived to predict different clinical outcomes using multivariate logistic regressions. In addition, chronic diseases included in the Charlson Comorbidity Index (CCI) and Elixhauser Comorbidity Index (ECI) were scored, and its accuracy of predicting COVID-19 clinical outcomes was also compared with the CCC.

Results: Data were retrieved from 90,549 ED patient visits during the study period, among which 3,864 patients were COVID-19 positive. Forty-seven point nine percent $(1,851 / 3,864)$ were admitted to the hospital, 9.4\% (364) patients were admitted to the ICU, 6.2\% (238) received invasive mechanical ventilation, and $4.6 \%$ (177) patients died in the hospital. The CCC evaluation correlated well with the four studied clinical outcomes. The adjusted odds ratios of predicting inhospital death from CCC was 2.84 (95\% confidence interval (CI): $1.81-4.45, \mathrm{P}<0.001)$. C-statistics of CCC predicting in-hospital all-

Manuscript submitted March 12, 2021, accepted March 26, 2021

Published online April 27, 2021

a Department of Emergency Medicine, JPS Health Network, 1500 S. Main St., Fort Worth, TX 76104, USA

bepartment of Information Technology, JPS Health Network, 1500 S. Main St., Fort Worth, TX 76104, USA

'These authors contributed equally to this article.

${ }^{\mathrm{d} C}$ Corresponding Author: Hao Wang, John Peter Smith Health Network, 1500

S. Main St., Fort Worth, TX 76104, USA. Email: hwang@ies.healthcare

doi: https://doi.org/10.14740/jocmr4476 cause mortality was $0.73(0.69-0.76)$, similar to those of the CCI's (0.72) and ECI's (0.71, $\mathrm{P}=0.0513)$.

Conclusions: CCC can accurately predict clinical outcomes among patients with COVID-19. Its performance accuracies for such predictions are not inferior to those of the CCI or ECI's.

Keywords: Clinical outcome; Comorbidity; COVID-19

\section{Introduction}

Currently, even with the efforts of social distancing, public masking policies, and appropriate disease management, coronavirus disease 2019 (COVID-19) has still spread widely in communities across the USA. By the end of 2020, in the USA alone, there were over 20 million COVID-19 patients with $340,000+$ associated deaths. COVID-19 has shown a variety of clinical outcomes including asymptomatic with no special treatment, symptomatic requiring hospitalization, intensive care unit (ICU) admissions requiring invasive mechanical ventilations (IMVs), and death [1-3]. Several risk factors predicting worse clinical outcomes have been reported and validated in many studies [4-6]. These risks include elderly, male, low socioeconomic status (SES), and comorbidities [7-10].

Previous studies have shown that comorbidities are predictive of worse clinical outcomes [11]. However, the comorbidities studied vary across studies with inconsistent findings $[12,13]$. At present, the most common COVID-related high-risk chronic conditions (CCCs) reported from different studies include hypertension, diabetes, chronic obstructive pulmonary disease (COPD), cancer, liver disease, chronic renal disease, and obesity [4, 14-16], among others. Given the fact that COVID-19 patients may have multiple comorbidities, it is important to understand the patterns of such comorbidities (e.g., number of comorbidities) in relation to clinical outcomes. Unfortunately, very few published studies have included such reports.

Apart from these individual studies, meta-analysis studies have also assigned different levels of risk (e.g., high, moderate, versus low) to comorbidities, leading to variation in the predicted clinical outcomes (e.g., ICU admission, hospital mortal- 
ity) [15, 17-19]. Different levels of risk are usually reported as pooled odds ratios (ORs) or risk ratios (RRs). However, these pooled OR/RRs were largely different. Nandy et al [20] reported a higher risk of chronic renal diseases than diabetes, higher risk of pulmonary disease than cardiovascular diseases to predict COVID-19 severity. However, Barek et al [21] reported a higher risk of diabetes than chronic renal disease, higher risk of cardiovascular diseases than pulmonary disease to predict COVID-19 severity. Barek et al [21] also found a higher risk of cancer than chronic renal disease predicting COVID-19 severity, whereas Ssentongo et al [22] found a higher risk of chronic renal disease than cancer for COVID-19 severity predictions. These differences raise the question of whether each CCC should be equally weighted for outcome predictions. Unfortunately, we were unable to find these answers in the current literature.

In previous reports, diverse comorbidities have been weighted differently to form a comorbidity index predicting disease severity and clinical outcomes (e.g., prolonged hospitalizations, hospital mortality) with Charlson Comorbidity Index (CCI), and Elixhauser Comorbidity Index (ECI)), and being used widely $[23,24]$. Both CCI and ECI used weighted comorbidities to predict disease severity among patients with chronic disease conditions. Under such circumstances, a performance accuracy comparison of weighted versus unweighted comorbidities to predict clinical outcomes could potentially provide answers on comorbidity evaluations among COVID-19 patients.

It is important to better understand the relationship between comorbidities and disease severity, particularly during the COVID-19 pandemic. Screening COVID-19 patients with comorbidities associated with unfavorable clinical outcomes could help prioritize disease management among this cohort, predict disease progress to allow appropriate medical resource allocation, and even help prevent disease by expediting the vaccination process. Therefore, in this study, we aim to: 1) determine the patterns of CCCs, especially on the association between the number of CCCs and four clinic outcomes (i.e., hospital admissions, ICU admissions, receiving IMV, and in-hospital mortality); and 2) further determine whether each CCC is equally weighted to predict disease clinic outcomes.

\section{Materials and Methods}

\section{Study design and setting}

This was a single-center retrospective observational study. The study hospital is an urban publicly funded hospital and a tertiary referral center with 573 licensed beds located in North Texas, USA. The study hospital Emergency Department (ED) is a level-one trauma center with approximately 120,000 annual patient visits. This study has been approved by the regional Institutional Review Board with waived informed consent (No. 1614030-1), and was conducted in compliance with the ethical standards of the responsible institution on human subjects.

\section{Study participants}

From March 1, 2020, to December 31, 2020, we screened all patients who presented to the study hospital ED. Among all these patients, we further screened for patients who had laboratory severe acute respiratory syndrome coronavirus 2 (SARS$\mathrm{CoV}$-2)-polymerase chain reaction (PCR) performed in the study ED or who had recently confirmed SARS-CoV-2-PCR tests done from outside facilities (within the past 14 days). We enrolled all patients who had positive SARS-CoV-2-PCR tests regardless of whether tests were done in the study ED or outside facilities. We excluded patients whose SARS-CoV-2-PCR tests were: 1) not done in the past 14 days from the index ED visits; or 2) negative.

\section{Data retrieval}

Study data were all retrieved from the electronic medical record (EMR) by two dedicated persons from the Department of Information Technology, who have received sufficient training on data management and who were initially blinded to this study (i.e., before the main results were open for all the individuals who participated in this project). We also randomly selected 20 patient datasets each time for three times from the entire dataset to manually check and validate the accuracy of data retrieval.

\section{Outcome measures}

Four clinical outcomes are measured including: 1) hospital admissions; 2) ICU admissions; 3) patients who received IMV during the hospitalizations; and 4) in-hospital all-cause mortality.

\section{Variables}

The key variables of this study are CCCs. We determined 11 CCCs based on: 1) previous literature reports associating chronic conditions with the severity of patient clinical outcomes (e.g., ICU admissions, mortalities, etc.) [7-9, 11, 12, 15, 16]; and 2) expert opinions using a modified Delphi's technique [25]. These CCCs include: 1) active cancer; 2) human immunodeficiency virus/acquired immunodeficiency syndrome (HIV/ AIDS); 3) chronic pulmonary disorders including COPD and asthma; 4) diabetes; 5) hypertension; 6) coronary artery diseases including myocardial infarction; 7) cerebrovascular accident (CVA); 8) chronic renal insufficiency/end-stage renal disease; 9) congestive heart failure; 10) liver cirrhosis; and 11) obesity. Obesity was defined as patient body mass index $(\mathrm{BMI}) \geq 30$, and other CCCs were defined by the International Classification of Disease, 10th version (ICD-10 code) used in the EMR. Meanwhile, general patient characteristics (age, gender, race/ethnicity) were also analyzed in this study. We divided age into four groups: 1) 18 - 54; 2) 55 - 60; 3) 61 - 65; and 4) 66 and older. Race/ethnicity is categorized based on the Federal Statistics and Program Administrative reporting of 
Table 1. Comparison of $\mathrm{CCC}, \mathrm{CCl}$, and ECl Comorbidity Evaluations

\begin{tabular}{llll}
\hline & CCC & CCI & ECI \\
\hline Active cancer & 1 & 2 & 7 \\
HIV/AIDS & 1 & 6 & 0 \\
Chronic obstructive pulmonary disease (COPD/asthma) & 1 & 3 & 3 \\
CHF & 1 & 3 & 9 \\
Hypertension & 1 & 1 & $0^{\mathrm{a}}$ \\
Coronary artery disease/myocardial infarction & 1 & 1 & $(0)$ \\
Cerebrovascular accident (CVA) & 1 & 5 \\
Diabetes & 1 & 3 & 0 \\
Obesity & 1 & $(0)$ & $0^{\mathrm{a}}$ \\
Chronic renal diseases & 1 & 2 & 6 \\
Liver cirrhosis & 1 & 3 & 4 \\
\hline
\end{tabular}

CCCs $(0-11)$ : each chronic condition is equally weighted. $\mathrm{CCl}$ : obesity not listed in $\mathrm{CCl}(0-25)$; ECl (AHRQ algorithm, 0 - 34): coronary artery disease/myocardial infarction not listed in ECI, HIVIAIDS and diabetes with no complication were listed as "0". "Obesity was scored as -5 and hypertension was scored as -1 in the original ECI AHRQ algorithm. However, due to previous report of risks of obesity and hypertension in COVID-19 patients, to avoid the offset effect of other CCCs, we scored obesity and hypertension as 0 in this study. CCC: COVID-related high-risk chronic condition; CCl: Charlson Comorbidity Index; ECl: Elixhauser Comorbidity Index; HIVIAIDS: human immunodeficiency virus/acquired immunodeficiency syndrome; CHF: congestive heart failure; AHRQ: Agency for Healthcare Research and Quality; COVID-19: coronavirus disease 2019.

basic racial and ethnic categories. We divided our patients into four groups: 1) non-Hispanic White (NHW); 2) non-Hispanic Black (NHB); 3) Hispanic/Latino (Hispanic); and 4) others. Due to the relatively small sample size of other race/ethnicities (including American Indian, Alaska Native, Asian, Native Hawaiian, or other Pacific Islanders, unknown, or patient refusal), we categorized these patients into one group.

\section{Study protocol}

First, we determined the association between the number of CCCs and different clinical outcomes. We classified patients with CCCs into four categories: 1) patients with no $\mathrm{CCC} ; 2$ ) patients with one CCC; 3) patients with two CCCs; and 4) patients with at least three CCCs. Second, we determined whether clinical outcomes for patients with CCCs of different categories can be predicted using a multivariate logistic regression model with the adjustment of other variables (i.e., age, gender, race/ethnicity). Third, to further determine whether each $\mathrm{CCC}$ should be weighted on the prediction of the severity of clinical outcomes, we measured the performance accuracy of CCC in comparison to those of the two comorbidity indexes. These two comorbidity indexes are CCI and ECI. Both have been used previously to predict the severity of disease and patient in-hospital mortality. Most of the CCCs are also included in the CCI and ECI. Each chronic condition in CCC is weighted equally, whereas each chronic condition in CCI and ECI is weighted differently (Table 1).

\section{Data analysis}

Analysis of variance (ANOVA) was used to compare clini- cal outcomes of different groups (e.g., patients with different CCCs). We used multivariate logistic regression analyses to determine patients with different categories of CCCs associated with four clinical outcomes with the adjustment of other variables (age, gender, race/ethnicity). Adjusted ORs (aORs) were reported with $95 \%$ confidence intervals (CI). Area under a receiver operating characteristic curve (AUC) was used to measure the performance accuracy of three different comorbidity evaluations (i.e., CCC, CCI, and ECI) predicting four clinical outcomes. STATA 16.0 (College Station, TX) was used for all study statistical analyses with $\mathrm{P}<0.05$ considered a statistically significant difference.

\section{Results}

From March 1, 2020, to December 31, 2020, we enrolled all ED patients who had laboratory confirmed positive SARSCoV-2-PCR tests (Fig. 1). Their general characteristics are listed in Table 2. We found that Hispanic patients had the highest positive SARS-CoV-2-PCR test rates (COVID-19 patients). Among all COVID-19 patients, Hispanic patients tend to be younger with female predominant $(\mathrm{P}<0.05)$, whereas NHW patients are male predominant. Four clinical outcomes were compared among different races/ethnicities. We found no statistically significant differences occurred (Table 2).

In this study, we focus on the number of CCCs that COVID-19 patients sustained and its relation to four clinical outcomes. In this cohort, nearly $40 \%$ of the COVID-19 patients $(1,509 / 3,864,39 \%)$ did not have CCCs. In contrast, $20 \%$ of COVID-19 patients had one CCC, 14\% had two CCCs, and $26 \%$ had at least three CCCs (Table 2). More importantly, the number of these CCCs is correlated to the severity of all four 


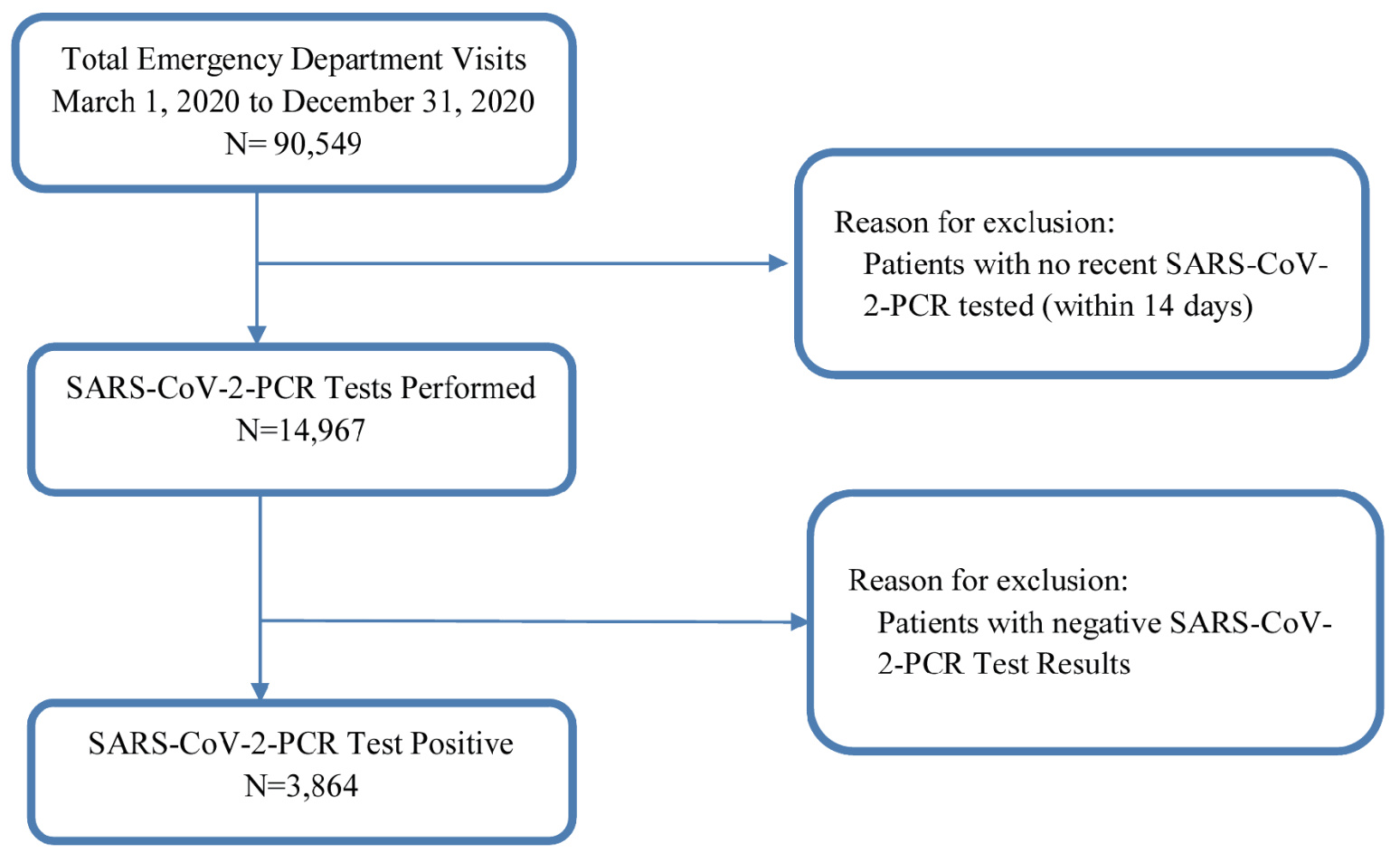

Figure 1. Study flow diagram. SARS-CoV-2: severe acute respiratory syndrome coronavirus 2; PCR: polymerase chain reaction.

clinical outcomes. The more CCCs patients sustained, the more severe of these clinical outcomes the patients could have $(\mathrm{P}<0.001$, Table 3$)$.

Furthermore, to better determine the relationship between CCCs and the severity of clinical outcomes, multivariate logistic regression was analyzed with the adjustments of all potential independent risks predicting disease clinical outcomes in the literature (e.g., age, gender, comorbidities, etc.). We found age and gender are two independent risks predicting these four clinical outcomes, whereas race/ethnicity is not (Table 4). Even with the adjustments of all potential confounders, the number of CCCs still predicts all four clinical outcomes independently (Table 4).

Finally, the AUCs of different comorbidity evaluation tools were compared to determine their performance accuracy. We found that similar performance accuracies of predicting four clinical outcomes among CCC, CCI, and ECI (Fig. 2 and Table 5). Therefore, it is unnecessary to weigh each CCC for

Table 2. General Characteristics of Study Patient Population

\begin{tabular}{|c|c|c|c|c|}
\hline & NHW & NHB & Hispanic & Others \\
\hline Patient visits, n (\%) & $29,410(32.5)$ & $30,022(33.2)$ & $26,109(28.8)$ & $5,008(5.5)$ \\
\hline Age (years), median (IQR) & $46(33,57)$ & $44(30,57)$ & $41(28,54)$ & $42(29,56)$ \\
\hline Number of COVID-19 tested at ED, $\mathrm{n}(\%)$ & $4,435(16.8)$ & $4,168(13.9)$ & $4,116(15.8)$ & $697(13.9)$ \\
\hline Number of COVID-19 positive, $\mathrm{n}(\%)^{*}$ & $794(15.3)$ & $1,103(24.7)$ & $1,711(37.5)$ & $256(33.9)$ \\
\hline Gender (male), n (\%)* & $463(58.3)$ & $540(49.0)$ & $826(48.3)$ & $127(49.6)$ \\
\hline Hospital admissions in COVID-19 patients, $\mathrm{n}(\%)$ & $393(49.5)$ & $519(47.1)$ & $823(48.1)$ & $116(45.3)$ \\
\hline ICU admissions among COVID-19 patients, n (\%) & $71(8.9)$ & $107(9.7)$ & $158(9.2)$ & $28(10.9)$ \\
\hline COVID-19 patients receiving ventilations, $\mathrm{n}(\%)$ & $47(5.9)$ & $65(5.9)$ & $107(6.3)$ & $19(7.4)$ \\
\hline
\end{tabular}

${ }^{*} \mathrm{P}<$ 0.05. NHW: non-Hispanic White; NHB: non-Hispanic Black; IQR: interquartile range; COVID-19: coronavirus disease 2019; ED: emergency department. 
Table 3. Association Between Clinical Outcome and Number of CCCs Among COVID-19 Patients

\begin{tabular}{|c|c|c|c|c|c|}
\hline & No $\mathrm{CCC}$ & One CCC & Two CCCs & $\geq 3 \mathrm{CCCs}$ & Pvalue \\
\hline Number of patients, $\mathrm{n}(\%)$ & $1,509(39)$ & $786(20)$ & $547(14)$ & $1,022(26)$ & \\
\hline Hospital admission, $\mathrm{n}(\%)$ & $559(37)$ & $373(47)$ & $275(50)$ & $644(63)$ & $<0.001$ \\
\hline Receiving mechanical ventilation, $\mathrm{n}(\%)$ & $42(2.8)$ & $43(5.5)$ & $45(8.2)$ & $108(10.6)$ & $<0.001$ \\
\hline All-cause in-hospital mortality, n (\%) & $32(2.1)$ & $29(3.7)$ & $29(5.3)$ & $87(8.5)$ & $<0.001$ \\
\hline
\end{tabular}

COVID-19: coronavirus disease 2019; CCC: COVID-related high-risk chronic condition.

clinical outcome predictions among COVID-19 patients.

\section{Discussion}

In this study, we find the number of CCCs is associated with all four clinical outcomes. Patients with more CCCs $(\geq 3)$ are associated with more severe COVID-19 clinical outcomes, which are independent of other risks (e.g., age and gender). In addition, it is unnecessary to weigh each CCC since their performance accuracy to predict clinical outcomes is not inferior to those of the CCI and ECI's. Our findings add extra evidence on the evaluation of COVID-19 severity among patients with multiple comorbidities. More importantly, it pro- vides a simple measurement on patients with comorbidities at risks of COVID-19, which can be used as a basic screening tool to prioritize disease prevention (e.g., recognizing susceptible patient populations), intervention (e.g., vaccination coverage), and management (e.g., allocating appropriate medical resources).

Our study had similar findings to previous reports in the literature $[26,27]$. We found clinical outcomes are similar regardless of race/ethnicity though more Hispanic patients were tested as positive. With the adjustment of age and gender, Hispanic patients had similar clinical outcomes in comparison to other racial/ethnic patients [28]. In addition, our study validated that age and gender are two independent risks predicting different clinical outcomes, similar to previous reports [7,29].

Table 4. The Adjusted Odds Ratios (aOR) of Number of CCCs, Age, Gender, and Racial/Ethnical Predictive of Four Different Clinical Outcomes

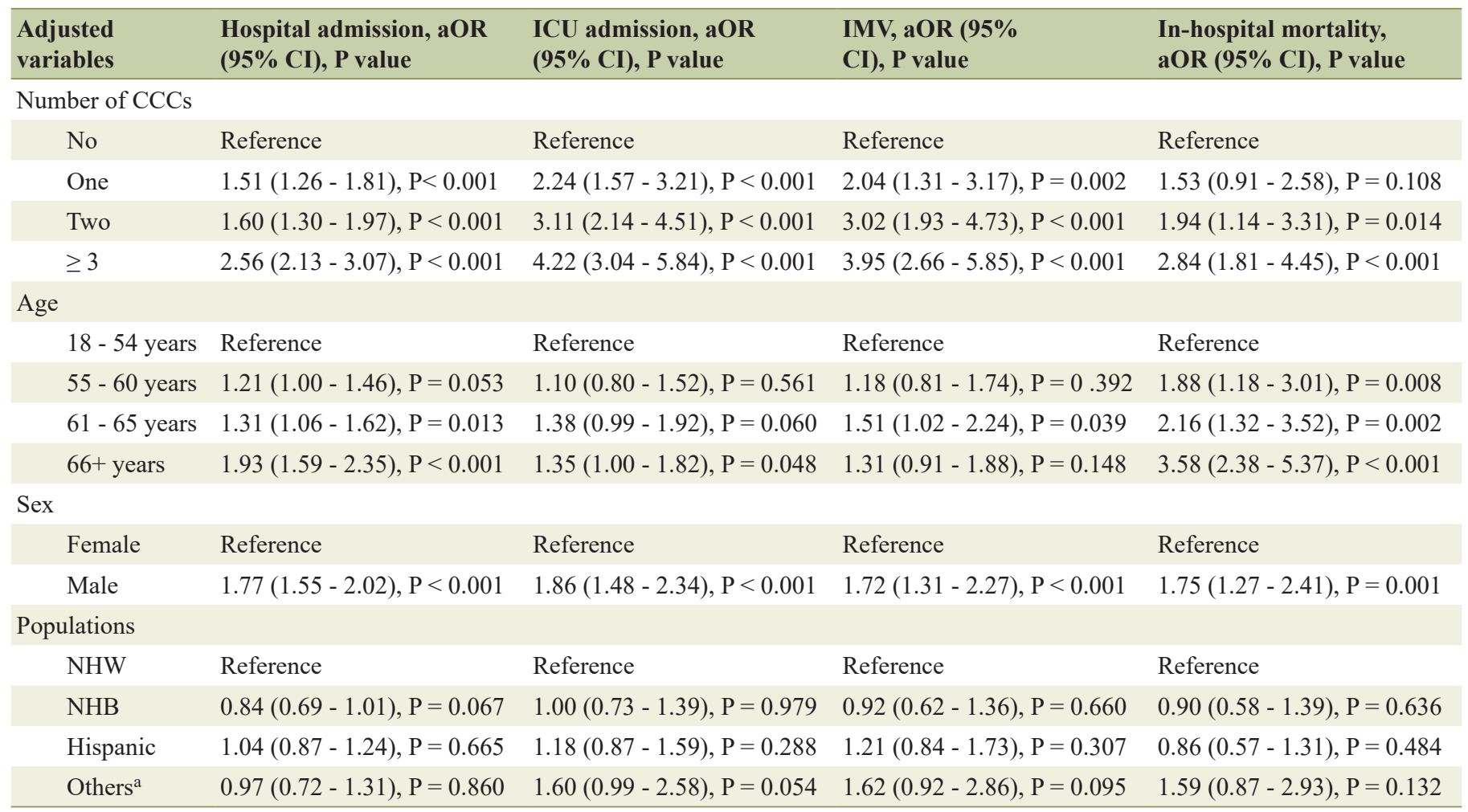

Cl: confidence interval; CCC: COVID-related high risk chronic condition; NHW: non-Hispanic White; NHB: non-Hispanic Black; ICU: intensive care unit; IMV: invasive mechanical ventilations. aOthers refer to American Indian, Alaska Native, Asian, Native Hawaiian or other Pacific Islanders, unknown, or patient refusal. 

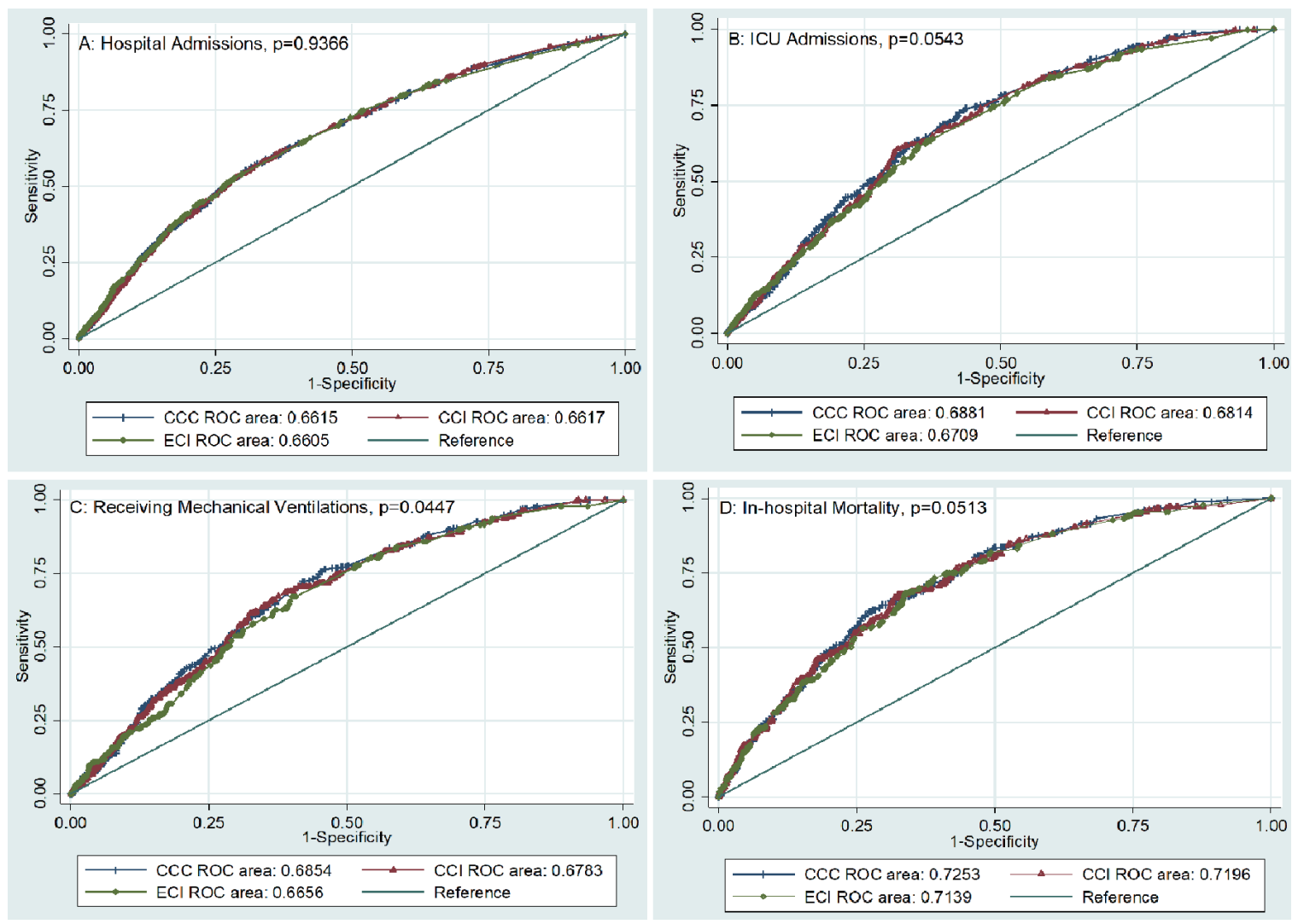

Figure 2. Using AUC to compare the performance accuracy of $\mathrm{CCC}, \mathrm{CCl}$, and $\mathrm{ECl}$ predictive of four different clinical outcomes. Panel A: Performance accuracy comparisons of hospital admission. Panel B: Performance accuracy comparisons of ICU. Panel C: Performance accuracy comparisons of patients receiving invasive mechanical ventilations. Panel D: Performance accuracy comparisons of in-hospital all-cause mortality. CCC: COVID-related high-risk chronic condition; CCI: Charlson Comorbidity Index; ECl: Elixhauser Comorbidity Index; ROC: receiver operating characteristics; ICU: intensive care unit; AUC: area under a receiver operating characteristic curve.

The difference of this study was to focus on the comorbidities of COVID-19 patients. While diverse CCCs have been reported previously $[7,11]$, we focused on these 11 chronic conditions due to relatively common occurrence among the study cohort. Using study CCC screening will help healthcare providers further prioritize COVID-19 disease prevention, evaluation, and management. Including too many chronic conditions would make such screening too complicated and less efficient. Similarly, screening too few comorbidities would miss significant amounts of patients with suboptimal sensitivity. Under this circumstance, we chose these 11 CCCs for further evaluation.
At present, we are uncertain whether each CCC should be weighted equally. Therefore, this study was performed to compare the performance accuracy of CCC, CCI, and ECI. $\mathrm{CCI}$ and ECI are both used widely to predict disease severity, prolonged hospitalization, and even in-hospital mortality with different weights on different chronic conditions [30, 31]. CCI and ECI have both been reported to accurately predict COVID-19 severity [32-34]. Therefore, we can use them as the reference to compare the performance accuracy of the study's simplified comorbidity evaluation (i.e., CCC). In addition, since we only used limited chronic conditions for such comparisons, it is not intended to confirm the superiority of using

Table 5. Using C-Statistics to Compare the Performance Accuracy of CCC, CCI, and ECI Predictive of Four Different Clinical Outcomes

\begin{tabular}{lllll} 
& CCC & CCI & ECI & P value \\
\hline Hospital admissions & $0.66(0.64-0.68)$ & $0.66(0.64-0.68)$ & $0.66(0.64-0.68)$ & 0.9366 \\
Intensive care unit admissions & $0.69(0.66-0.71)$ & $0.68(0.66-0.71)$ & $0.67(0.64-0.70)$ & 0.0543 \\
Receiving mechanical ventilations & $0.69(0.65-0.72)$ & $0.68(0.65-0.71)$ & $0.67(0.63-0.70)$ & 0.0447 \\
In-hospital mortality & $0.73(0.69-0.76)$ & $0.72(0.68-0.76)$ & $0.71(0.68-0.75)$ & 0.0513 \\
\hline
\end{tabular}

CCC: COVID-related high-risk chronic condition; CCl: Charlson Comorbidity Index; ECl: Elixhauser Comorbidity Index. 
CCC instead of CCI/ECI for overall accuracy prediction of disease severity. Our study only proved that equally weighted $\mathrm{CCC}$ function as the same as CCI/ECI for COVID-19 clinical outcome predictions. A future study is warranted to further determine the performance accuracy of $\mathrm{CCI} / \mathrm{ECI}$ predicting disease severity with all chronic conditions being evaluated.

Our study has its limitations. The first is patient selection bias because incomplete/missing/incorrect data cannot be avoided due to the nature of retrospective single-center study design. Second, we only included 11 chronic conditions in this study. However, other chronic conditions reported to be associated with COVID-19 disease severity are not included. This may affect the overall accuracy of this model prediction. Third, we included patients with $\geq 3$ CCCs into one category. Using such a category may affect the power of the study since we do not know whether the number of comorbidities still correlated well with the disease severity in patients with more than three comorbidities. However, such patients $(\geq 3$ comorbidities) might account for very few numbers of patients thus the performance accuracy of disease severity predictions might be less affected. Fourth, this study only includes age, gender, and race/ethnicity as potential independent risks affecting COVID-19 disease severity in the multivariate logistic regression model, other potential risks predicting disease severity are not included, which may affect the final aORs of comorbidities. Therefore, a large-scale prospective multi-center study is warranted for further validations.

\section{Conclusions}

Patients with an increased number of CCCs tended to have increased risks of hospital admissions, ICU admissions, receiving IMV, and in-hospital all-cause mortality. Such risks are independent and can be equally weighted to predict clinical outcomes.

\section{Acknowledgments}

We thank Radhika Cheeti from Informational Technology Department for study data retrieval and validations.

\section{Financial Disclosure}

There was no specific funding source to be mentioned.

\section{Conflict of Interest}

The authors declare that they have no conflict of interest.

\section{Informed Consent}

This study was approved by local Institutional Review Board with waived informed consent.

\section{Author Contributions}

JJK, SS, DPB, and HW have designed and performed the study. JJK, DPB, AFH, JPD, CDS, and HW have drafted the manuscript and did critical editing. SS, DPB, and HW have assisted and supported in sample collection and subsequent analysis with statistics. JJK, SS, AFH, CDS, and HW have carefully supervised this manuscript preparation and writing.

\section{Data Availability}

The authors declare that data supporting the findings of this study are available upon requesting to the correspondence author.

\section{References}

1. Buckner FS, McCulloch DJ, Atluri V, Blain M, McGuffin SA, Nalla AK, Huang ML, et al. Clinical features and outcomes of 105 hospitalized patients with COVID-19 in Seattle, Washington. Clin Infect Dis. 2020;71(16):21672173.

2. Gomez-Ochoa SA, Franco OH, Rojas LZ, Raguindin PF, Roa-Diaz ZM, Wyssmann BM, Guevara SLR, et al. COVID-19 in health-care workers: a living systematic review and meta-analysis of prevalence, risk factors, clinical characteristics, and outcomes. Am J Epidemiol. 2021;190(1):161-175.

3. Lai CC, Liu YH, Wang CY, Wang YH, Hsueh SC, Yen MY, Ko WC, et al. Asymptomatic carrier state, acute respiratory disease, and pneumonia due to severe acute respiratory syndrome coronavirus 2 (SARS-CoV-2): Facts and myths. J Microbiol Immunol Infect. 2020;53(3):404412.

4. Guan WJ, Ni ZY, Hu Y, Liang WH, Ou CQ, He JX, Liu $\mathrm{L}$, et al. Clinical Characteristics of Coronavirus Disease 2019 in China. N Engl J Med. 2020;382(18):1708-1720.

5. Li B, Yang J, Zhao F, Zhi L, Wang X, Liu L, Bi Z, et al. Prevalence and impact of cardiovascular metabolic diseases on COVID-19 in China. Clin Res Cardiol. 2020;109(5):531538.

6. Bello-Chavolla OY, Bahena-Lopez JP, Antonio-Villa NE, Vargas-Vazquez A, Gonzalez-Diaz A, Marquez-Salinas A, et al. Predicting mortality due to SARS-CoV-2: a mechanistic score relating obesity and diabetes to COVID-19 outcomes in Mexico. J Clin Endocrinol Metab. 2020;105:2752-2761.

7. Fang X, Li S, Yu H, Wang P, Zhang Y, Chen Z, Li Y, et al. Epidemiological, comorbidity factors with severity and prognosis of COVID-19: a systematic review and metaanalysis. Aging (Albany NY). 2020;12(13):12493-12503.

8. Porzionato A, Emmi A, Barbon S, Boscolo-Berto R, Stecco C, Stocco E, Macchi V, et al. Sympathetic activation: a potential link between comorbidities and COVID-19. FEBS J. 2020;287(17):3681-3688.

9. Iavarone M, D'Ambrosio R, Soria A, Triolo M, Pugliese 
N, Del Poggio P, Perricone G, et al. High rates of 30day mortality in patients with cirrhosis and COVID-19. J Hepatol. 2020;73(5):1063-1071.

10. Mattos Dos Santos R. Isolation, social stress, low socioeconomic status and its relationship to immune response in Covid-19 pandemic context. Brain Behav Immun Health. 2020;7:100103.

11. Guan WJ, Liang WH, Zhao Y, Liang HR, Chen ZS, Li YM, Liu XQ, et al. Comorbidity and its impact on 1590 patients with COVID-19 in China: a nationwide analysis. Eur Respir J. 2020;55(5).

12. Grasselli G, Greco M, Zanella A, Albano G, Antonelli M, Bellani G, Bonanomi E, et al. Risk Factors Associated With Mortality Among Patients With COVID-19 in Intensive Care Units in Lombardy, Italy. JAMA Intern Med. 2020;180(10):1345-1355.

13. Garcia-Pachon E, Zamora-Molina L, Soler-Sempere MJ, Baeza-Martinez C, Grau-Delgado J, Padilla-Navas I, Gutierrez F. Asthma and COPD in hospitalized COVID-19 patients. Arch Bronconeumol. 2020;56(9):604-606.

14. Kuderer NM, Choueiri TK, Shah DP, Shyr Y, Rubinstein SM, Rivera DR, Shete S, et al. Clinical impact of COVID-19 on patients with cancer (CCC19): a cohort study. Lancet. 2020;395(10241):1907-1918.

15. Oyelade T, Alqahtani J, Canciani G. Prognosis of COVID-19 in patients with liver and kidney diseases: an early systematic review and meta-analysis. Trop Med Infect Dis. 2020;5(2).

16. Busetto L, Bettini S, Fabris R, Serra R, Dal Pra C, Maffei P, Rossato M, et al. Obesity and COVID-19: An Italian Snapshot. Obesity (Silver Spring). 2020;28(9):16001605.

17. Desai A, Sachdeva S, Parekh T, Desai R. COVID-19 and cancer: lessons from a pooled meta-analysis. JCO Glob Oncol. 2020;6(557-559.

18. Cooper TJ, Woodward BL, Alom S, Harky A. Coronavirus disease 2019 (COVID-19) outcomes in HIV/AIDS patients: a systematic review. HIV Med. 2020;21(9):567577.

19. Hussain A, Mahawar K, Xia Z, Yang W, El-Hasani S. Obesity and mortality of COVID-19. Meta-analysis. Obes Res Clin Pract. 2020;14(4):295-300.

20. Nandy K, Salunke A, Pathak SK, Pandey A, Doctor C, Puj $\mathrm{K}$, Sharma M, et al. Coronavirus disease (COVID-19): A systematic review and meta-analysis to evaluate the impact of various comorbidities on serious events. Diabetes Metab Syndr. 2020;14(5):1017-1025.

21. Barek MA, Aziz MA, Islam MS. Impact of age, sex, comorbidities and clinical symptoms on the severity of COVID-19 cases: A meta-analysis with 55 studies and 10014 cases. Heliyon. 2020;6(12):e05684.

22. Ssentongo P, Ssentongo AE, Heilbrunn ES, Ba DM, Chinchilli VM. Association of cardiovascular disease and
10 other pre-existing comorbidities with COVID-19 mortality: A systematic review and meta-analysis. PLoS One. 2020;15(8):e0238215.

23. Moore BJ, White S, Washington R, Coenen N, Elixhauser A. Identifying increased risk of readmission and in-hospital mortality using hospital administrative data: the AHRQ elixhauser comorbidity index. Med Care. 2017;55(7):698-705.

24. Quan H, Li B, Couris CM, Fushimi K, Graham P, Hider $\mathrm{P}$, Januel JM, et al. Updating and validating the Charlson comorbidity index and score for risk adjustment in hospital discharge abstracts using data from 6 countries. Am J Epidemiol. 2011;173(6):676-682.

25. McMillan SS, King M, Tully MP. How to use the nominal group and Delphi techniques. Int J Clin Pharm. 2016;38(3):655-662.

26. Webb Hooper M, Napoles AM, Perez-Stable EJ. COVID-19 and racial/ethnic disparities. JAMA. 2020;323(24):24662467.

27. Price-Haywood EG, Burton J, Fort D, Seoane L. Hospitalization and mortality among black patients and white patients with COVID-19. N Engl J Med. 2020;382(26):25342543.

28. Rentsch CT, Kidwai-Khan F, Tate JP, Park LS, King JT, Jr., Skanderson M, Hauser RG, et al. Patterns of COVID-19 testing and mortality by race and ethnicity among United States veterans: A nationwide cohort study. PLoS Med. 2020;17(9):e1003379.

29. Jin JM, Bai P, He W, Wu F, Liu XF, Han DM, Liu S, et al. Gender differences in patients with COVID-19: focus on severity and mortality. Front Public Health. 2020;8:152.

30. Austin SR, Wong YN, Uzzo RG, Beck JR, Egleston BL. Why summary comorbidity measures such as the charlson comorbidity index and elixhauser score work. Med Care. 2015;53(9):e65-72.

31. Simard M, Sirois C, Candas B. Validation of the Combined comorbidity index of charlson and elixhauser to predict 30-day mortality across ICD-9 and ICD-10. Med Care. 2018;56(5):441-447.

32. Christensen DM, Strange JE, Gislason G, Torp-Pedersen C, Gerds T, Fosbol E, Phelps M. Charlson comorbidity index score and risk of severe outcome and death in Danish COVID-19 patients. J Gen Intern Med. 2020;35(9):28012803.

33. Zhou W, Qin X, Hu X, Lu Y, Pan J. Prognosis models for severe and critical COVID-19 based on the Charlson and Elixhauser comorbidity indices. Int J Med Sci. 2020;17(15):2257-2263.

34. Tuty Kuswardhani RA, Henrina J, Pranata R, Anthonius Lim M, Lawrensia S, Suastika K. Charlson comorbidity index and a composite of poor outcomes in COVID-19 patients: A systematic review and meta-analysis. Diabetes Metab Syndr. 2020;14(6):2103-2109. 